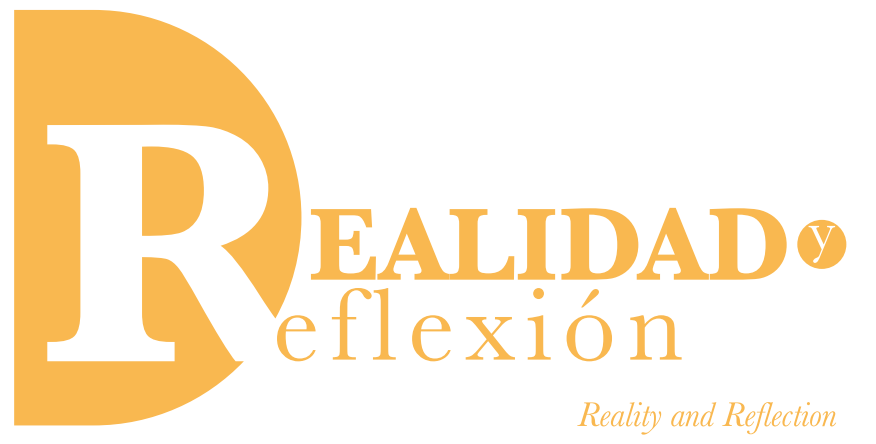

ISSN 1992-6510

Año 18, N 48, San Salvador, El Salvador, Centroamérica. Revista Semestral Julio-Diciembre 2018

YEAR 18, N 48, SAN SALVADOR, EL SALVADOR, CENTRAL AMERICA. SEMESTRAL JouRnaL JULY-DECEMBER 2018

\title{
Importancia de la construcción del Pensamiento Crítico en el proceso de elaboración de trabajos académicos en la Educación Superior
}

\section{Importance of the construction of Critical Thinking in the process of preparing academic paper in higher education}

\author{
Daniel López Fuentes \\ Maestro en Métodos y Técnicas de Investigación Social \\ Docente de la Universidad Francisco Gavidia \\ dlopez@ufg.edu.sv \\ Glenda Yamileth Trejo Magaña \\ Maestra en Metodología de la Investigación Científica \\ Docente de la Universidad de Sonsonate \\ gtrejo@usonsonate.edu.sv \\ Fecha de recepción: 30 de septiembre de 2018 \\ Fecha aprobación: 17 de diciembre de 2018
}

\section{RESUMEN}

Este artículo tiene como finalidad exponer la importancia de la construcción del Pensamiento Crítico en el proceso de elaboración de trabajos académicos en la Educación Superior. Se define como la habilidad que capacita al estudiante para hacer preguntas y juicios de valor; de tal manera que es una forma superior del pensamiento. Esta forma de abordaje de los problemas posibilita la generación de nuevas formas de pensar y actuar de manera amplia y estratégica, formulando conocimientos nuevos sin necesidad de seguir manuales, formatos preestablecidos y sobretodo siguiendo su propio razonamiento. Y por último, gracias a esta forma de pensar permite el empoderamiento intelectual para poder en último término transformar la realidad.

Palabras clave: pensamiento crítico, trabajos académicos, educación superior, universidad, docentes y estudiantes.

ABSTRACT

The purpose of this article is to expose the importance of the construction of Critical Thinking in the process of elaboration of academic works in Higher Education. It is defined as the skill that enables the student to ask questions and value judgments; in such a way that it is a superior form of thought. 
This way of approaching problems enables the generation of new ways of thinking and acting in a broad and strategic manner, formulating new knowledge without the need to follow manuals, pre-established formats and, above all, following their own reasoning. And finally, thanks to this way of thinking allows intellectual empowerment to ultimately transform reality.

Keywords:critical thinking, academic papers, Higher Education, University, Teachers and Students.

A modo de desarrollo teórico se entiende el Pensamiento Crítico como una habilidad que involucra lo holístico, en el sentido que se logra capacitar al estudiante, para desmenuzar la información por medio de preguntas, juicios analíticos y comprensión de conceptos y definiciones, esto gracias a la argumentación y búsqueda de solución de un problema; por medio de la indagación de su razón medular de existir, es decir la comprensión de su Yo interno frente al contexto social que lo rodea (Facione, 2007).

Se debe tener en cuenta que el Pensamiento Crítico es una capacidad de pensar de manera más clara, reflexiva y sobretodo con una independencia ideológica, lo cual a la postre le permite a la persona critica crear juicios de valor fiables y afirmados en la realidad tangible. De ahí que, en materia de Educación Superior se sostiene que la habilidad de pensar críticamente tiene un modelo a seguir y una estructura que permite ordenar ideas, tener una manera de actuar acorde a la moralidad y valores necesarios para formar una visión de país; con la diferencia a otras formas de pensamiento pues estaría basado en pruebas académicas (Águeda, 2011).

Por otro lado, gracias al Pensamiento Crítico los estudiantes podrán analizar las fuentes de información existentes en sus entornos; ya que en este S.XXI, la dificultad radica en discernir de la masividad de información y no en su localización o búsqueda, por tanto con el Pensamiento Crítico se da la posibilidad de clasificar por niveles de calidad, refutabilidad, peso académico y demás elementos que conviertan los trabajos redactados por los estudiantes en verdaderas fuentes secundarias de consulta y conocimiento, gracias a la fiabilidad de la información y los argumentos lógicos, coherentes y bien fundamentados (El Educador, 2008). A la vez al desarrollar verdaderos trabajos académicos tipo ensayo argumentativo se llegará a evaluar la realidad por medio de la presentación de conclusiones concisas y precisas formuladas por ellos mismos y apoyados en datos, fuentes y evidencias objetivas de la realidad.

Otro de los retos para los estudiantes en este Siglo, se centra en la necesidad de formar y desarrollar la habilidad de actuar y desentrañar el mundo real que los rodea, puesto que es ineludible inferir que el mundo académico y laboral está en constante cambio. Por tanto la toma de decisiones aplicando el Pensamiento Crítico es de vital importancia para la educación y vida universitaria y laboral, logrando convertirse en un engranaje productivo de la sociedad salvadoreña.

Por esta razón las experiencias educativas en la Educación Superior deberían tener como misión el hecho de capacitar en el proceso de análisis crítico de la realidad y eso pasa necesariamente por capacitar al cuerpo docente para que ellos trabajen estas habilidades con sus estudiantes, de esta manera los productos de las clases, de los trabajos de graduación y cualquier contarán con 
la rigurosidad de un marco analítico en función de la población y realidad salvadoreña.

Siguiendo la idea, se debe de ver el Pensamiento Crítico como la variedad de capacidades intelectuales sumadas a la buena disposición de darle al país una serie de aportes académicos a través de los trabajos de los estudiantes universitarios, esto traería el beneficio y retribución social que devuelva el protagonismo a los estudiantes que se están formando en las distintas casas del saber y que por ahora no se piensa necesariamente en generar los espacios para que den soluciones a los problemas de país más urgentes. Ahora bien, es importante tener claro que el estudiante universitario debe estar preparado para los retos académicos y la necesidad de poder sopesar la masividad de la información que se encuentra en el internet. De ahí que una de las primeras habilidades sea conocer acerca de las Tecnologías de la Información y Comunicación, a la vez poseer las herramientas que da la Sociedad de la Información (Cubas, 2012).

Esto se debe al hecho de que como se mencionó ya, la dificultad actual no es de información, sino de tener los criterios adecuados para procesar y ponderarla con el fin de entenderla y aplicarla con un sentido de pertenencia y rigurosidad, permitiendo la certeza de que se escribe con propiedad y se aplica a la realidad cambiante e incierta. Esto significa que el S.XXI trae en estas casi dos décadas un escenario volátil, incierto y complejo, los cuales se pueden entender fundamentándose en el Pensamiento Crítico, favoreciendo la posibilidad de asumir una postura o una posición académica de la realidad y no basado solo en lo ideológico o mediático (Gerras, 2006).
Por lo tanto, se espera que los estudiantes de Educación Superior tengan las capacidades, habilidades y fortalezas para lidiar con la realidad que los rodea y que viven constantemente, así es como se logrará una independencia de pensamiento y empoderamiento intelectual, evitando caer en el pensamiento vano y sin fundamentos; así es también como se analizará la realidad y se tendrá la capacidad de generar conclusiones y recomendaciones ante la situación coyuntural o estructural salvadoreño (Paul \& Elder, 2003). La idea de esto es llegar al autoconocimiento y autodeterminación del estudiante empoderándose realmente de su profesión para aplicarlo al contexto socio económico y político que vive, no solo para resolver el corto plazo, sino crear una visión de país a mediano y largo plazo que aglutine al resto de sociedad salvadoreña.

Surge la pregunta ¿̇Para qué le sirve al cuerpo estudiantil las herramientas del Pensamiento Crítico? Fundamentalmente es para sostener una posición frente a la realidad, la cual estará fundamentada en la capacidad de poseer un juicio propio y analítico fundamentado en la capacidad de captar el problema medular de una determinada realidad y darle un tratamiento para solucionarlo basado en la interpretación holística de las causas y consecuencias que lo forman, logrando generar las alternativas idóneas para superar los obstáculos académicos y de la vida, lo que se espera es que la juventud-que ya es el futuro salvadoreñotome decisiones que permitan un progreso a $\mathrm{El}$ Salvador y sobretodo generar una real visión de país (Paul \& Elder, 2005).

Para lograrlo es necesario ver el rol de las instituciones de Educación Superior; como 
primer punto estas organizaciones poseen un proyecto, visión y misión educativas definidas, las cuales se manifiestan en el último eslabónque es el docente y que impregnan al estudiante en los salones de clase-por tanto se necesita que ambos actores fomentan y mantengan una educación continua, teniendo en cuenta que la educación evoluciona día a día, de ahí que los paradigmas educativos no deben enquistarse en el cuerpo docente; por tanto de manera autodidacta o por medio de las autoridades se necesita que existe una capacitación, monitoreo y actualización en las ramas que el cuerpo docente desarrolla, con el fin de transmitirlo al estudiantado, el cual lo deberá reflejar en los trabajos académicos que poseerán rigor y Pensamiento Crítico (Saladino, 2012).

Este proceso se debe hacer así, pues, en primer lugar, es la manera en que se sistematiza el empoderamiento académico, el cual llevará a la utilización de habilidades laborales en la vida profesional; segundo, el estudiantado podrá aplicar el pensamiento democrático a través de la resolución de conflictos de manera respetuosa al discutir las ideas de manera propositiva y analítica, para llegar a una conclusión y toma de decisiones optima que le sirva de manera personal y colectiva y tercero, por medio de la aplicación del Pensamiento Crítico en los trabajos académicos de nivel superior, el estudiantado evolucionará en su forma de pensar y ver la realidad, a la vez tendrá la capacidad de socializar frente a sus pares y docentes los conocimientos y así aplicarlos a la realidad objetiva y subjetiva $y$, por último, de esta manera, el cuerpo estudiantil, por medio de la redacción de trabajos académicos aplicando el Pensamiento Crítico, le permitirá crear parámetros idóneos en el análisis de su realidad $y$ contexto con el fin de transformarla si fuera el caso necesario, dicho cambio se lograría por medio de un sistemático proceso de validación y de ideas y posturas (Cubas, 2012).

Por estas cuatro razones la idea de la aplicación del Pensamiento Crítico consiste en formar verdaderos líderes transformacionales capaces de formar posturas y conclusiones frente a su realidad superando los estándares intelectuales por medio de la resolución de conflictos de una manera democrática, participativa y activa.

Bajo este análisis se comprende que el Pensamiento Crítico en los trabajos académicos deberán mostrar un conocimiento sustentado en fuentes válidas, con el fin de llegar a conclusiones aplicables. Ante eso el estudiantado tendrá la capacidad de regular su propio conocimiento e ideas con una base conceptual, comprobable y sobretodo contextualizada con su realidad y aplicando la sistematización de las ideas por medio de una disciplina académica y metodológica (Campos, 2007).

En ese sentido el Pensamiento Crítico está relacionado con la visión de país, pues se formará y contextualizará la realidad con base a preguntas que busquen analizar y comprender la esencia, pero sobre todas las cosas la capacidad transformacional de la realidad por medio del rigor académico. Por tal razón se comprende que el Pensamiento Crítico servirá como una habilidad de determinar y comprender las debilidades y fortalezas que cada estudiante tenga, y así formar una disciplina académica a la hora de analizar la realidad y ponderar la veracidad de postulados que se le presentan sin mayor explicación. 
Ahora bien ¿Cuáles son los beneficios del Pensamiento Crítico que pueden aplicarse en la redacción de trabajos académicos en la Educación Superior? En primer lugar fomentará y fortalecerá el proceso de enseñanza y de aprendizaje, segundo el estudiantado al analizar su realidad destacará ideas principales para crear un juicio académico frente a la realidad mutable, tercero la honestidad intelectual, que tanto hace daño en la redacción de artículos y tareas, cuarto poseer una mente amplia basado en la eliminación de dogmas estructuras mentales y paradigmas, lo cual permitirá la formación de un análisis riguroso de la realidad, por medio de la eliminación de sesgos de pensamiento y así encontrar la veracidad de su entorno a través de los juicios razonados y esquematizados (Paul \& Elder, La Mini-guía para el Pensamiento Crítico, 2003).

Este proceso de esquematizar ideas se logra a partir de formular una idea principal que se sustenta con argumentos fundamentados en fuentes bibliográficas sólidas y un análisis de la realidad contextual, convirtiendo al estudiante en una persona responsable y genuina tanto en la redacción original de su trabajo académico, como en los análisis e interpretación académica que haga de su realidad (Gerras S. , 2010).

Siguiendo el hilo conductor se entiende que las destrezas del Pensamiento Crítico están regidas por lo intelectual y lo personal, es así como este binomio mente y contexto deberían convertir y formar un razonamiento progresista y constante. Lo cual se logra gracias al compromiso de esforzarse por captar su entorno por medio de sopesar los diversos puntos de vista y escoger las posiciones que estén sustentadas en la argumentación (Campos, 2007).
Entonces ¿̇Para qué sirve el Pensamiento Crítico? Lo fundamental es para cuestionar la realidad que rodea al cuerpo estudiantil universitario, también para comprender que de manera individual se pueden hacer transformaciones a nivel macro dentro de la sociedad, pues se emiten juicios de valor que van más allá de los preestablecidos, por terceros, pues el Pensamiento Crítico tiene la funcionabilidad de evaluar, razonar y preparar al estudiante en la toma de decisiones más idónea frente al cumulo de estereotipos, subjetividades y estigmas que lo rodean, siempre aplicando la tan importante honestidad intelectual, propia de la academia.

Todo esto servirá para matizar y traslapar las fuentes de información, creando un razonamiento reflexivo, con el fin de razonar los argumentos propuestos y mantener la coherencia intelectual, por medio de la secuencia lógica de evidencias que sustenten las afirmaciones o la tesis a defender por el estudiantado (Gerras S. , 2006).

A manera de conclusión, el Pensamiento Crítico le permitirá a los estudiantes sostener sus ideas con argumentos válidos y defendibles, pues superaran el conocimiento básico y común, a la vez se desecharán las falacias y demás sesgos del pensamiento pues todo se sustentará con fuentes confiables y verificables.

E1 Pensamiento Crítico permitirá cuestionarse ante los temas preestablecidos y las realidades sociales, gracias a que para el estudiante serán temas de interés, ya que con la información que tenga, entenderá y aplicará soluciones, con el fin de incidir en su entorno mostrando conclusiones basadas en premisas intelectuales. 


\section{Citas Bibliográficas}

Águeda, B. (2011). Nuevas claves para la Docencia Universitaria en el espacio Europeo de Educación Superior. Madrid: Narcea.

Campos, A. (2007). Pensamiento Crítico. Técnicas para su desarrollo. Bogotá: Magisterio.

Cubas, M. (2012). ¿Cómo desarrollar un Pensamiento Crítico? México DF: Universidad Panamericana México.

El Educador. (2008). Pensamiento Crítico. Lima: Grupo editorial Norma.

Facione, P. (2007). Pensamiento Crítico: ¿Qué es y por qué es importante? California: The California Academic Press.
Gerras, S. (2006). Pensando críticamente a cerca del Pensamiento Crítico: Guía Fundamental para Líderes Estratégicos. Carlisle: Army War College.

Gerras, S. (2010). ¿Cómo desarrollar pensadores creativos y críticos? Pensilvania: Military Review. Paul, R., \& Elder, L. (2003). La Mini-guía para el Pensamiento Crítico. California: Fundación para el Pensamiento Crítico.

Paul, R., \& Elder, L. (2005). Estándares de competencia para el Pensamiento Crítico. California: Fundación para el pensamiento Crítico.

Saladino, A. (2012). Pensamiento Crítico. México DF: UNAM. 